\title{
Morphologic and temporal characterisation of lesions in an enhanced murine model of Serpulina hyodysenteriae infection
}

\author{
D. L. HUTTO ${ }^{\S}$, J. E. GALVIN* and M. J. WANNEMUEHLERं
}

Department of Veterinary Pathology, College of Veterinary Medicine, lowa State University, Ames, IA, * Department of Microbiology and Immunology, University Health Sciences Center, Univeristy of Oklahoma, Oklahoma City, OK and †Department of Microbiology, Immunology and Preventive Medicine, College of Veterinary Medicine, lowa State University, Ames, IA, USA

This laboratory has previously reported a murine model of Serpulina hyodysenteriae infection in which mice fed a defined diet, Teklad 85420 (TD), developed caecal lesions more consistently than mice fed a conventional rodent chow (CRC). The objectives of the current studies were to characterise and compare the time of onset of lesions, the morphological nature and severity of lesions and the extent of colonisation by $S$. hyodysenteriae in mice fed the two diets. In the first of two experiments, $50 \mathrm{C} 3 \mathrm{H} / \mathrm{HeJ}$ and $50 \mathrm{C3H} / \mathrm{HeOuJ}$ mice were fed either TD or CRC and then half of each group was infected with $S$. hyodysenteriae. Mice $(n=5)$ from each group were killed and examined on days, 1, 2, 4, 9 or 17 after infection. Each mouse was examined grossly and microscopically and assigned lesion scores based on lesion severity. The second experiment was designed in an identical way to the first, but had slightly smaller group sizes $(n=20)$. Mice $(n=4)$ were killed for necropsy at the same five time points after infection and their caeca were homogenised and examined by quantitative bacteriology with media selective for $S$. hyodysenteriae. There were no differences in any finding due to mouse strain. Group lesion scores over the entire experimental period were significantly higher in mice fed TD (mean total lesion index $=13$ ) than in mice fed CRC (mean total lesion index $=8.8$ ). Lesions were also temporally distributed in a significantly different manner in that they appeared earlier (day 1) and persisted longer in the TD-fed mice in comparison to CRC-fed mice. Furthermore, lesions of equivalent severity from each treatment group presented identical microscopic features. Finally, quantitative bacteriological results indicated that there was no significant difference in the number of cfu of $S$. hyodysenteriae isolated from mice fed TD and those fed CRC. These results demonstrate that the characteristic severe lesions associated with $S$. hyodysenteriae infection in mice can occur 1 day after oral challenge in mice fed Teklad diet $\mathbf{8 5 4 2 0}$. Bacteriological results further indicate that the enhancement of lesion formation in this model is not due to any significant effect of the diet on numbers of spirochaetes in the caeca of infected mice.

\section{Introduction}

Serpulina hyodysenteriae, a large gram-negative spirochaete, is the aetiological agent of swine dysentery, a highly transmissible diarrhoeal disease of growing swine. Swine dysentery is characterised grossly by a mucohaemorrhagic caecitis and colitis and microscopically by superficial epithelial cell necrosis, lamina

Received 15 May 1997; accepted 15 July 1997.

Corresponding author: Dr M. J. Wannemuehler.

${ }^{\S}$ Present address: Department of Biomedical Sciences, Tufts School of Vetinary Medicine, N. Grafton, MA, 01536, USA. proprial congestion and inflammatory cell infiltration, and colonic gland hyperplasia. Spirochaetes are associated with the mucosa at the luminal surface and within the colonic glands [1-3]. Numerous species of small laboratory animals have been infected experimentally with $S$. hyodysenteriae, including mice, guinea-pigs and chickens. The mouse model has been the most extensively used, both in enteropathogenicity testing of different strains of $S$. hyodysenteriae and in studies on pathogenesis [4-11]. Lesions in mice are confined to the caecum, and are characterised grossly by caecal atrophy and mural oedema. Microscopic lesions in mice consist of superficial epithelial cell necrosis, colonic 
gland elongation and lamina proprial and submucosal inflammatory cell infiltrates and oedema $[4,6,11]$. This laboratory has reported on strain-related differential susceptibility in mice to $S$. hyodysenteriae infection and on the use of a defined diet (Teklad diet 85420) to enhance the susceptibility of mice to the pathogenic effects of $S$. hyodysenteriae infection $[8,12]$. It has been noted that susceptible mice fed this diet may develop gross lesions within $24 \mathrm{~h}$ of infection. In contrast, in most studies utilising mice as an infection model for swine dysentery, principals are killed and examined 720 days after infection. The purposes of these experiments were to characterise the morphological changes that occur in this enhanced murine model of $S$. hyodysenteriae infection and to compare their severity and occurrence over time with those same changes in the conventional murine model of $S$. hyodysenteriae infection. In addition, bacteriological studies were performed to ascertain if demonstrated differences in lesion formation could be attributed to the extent of $S$. hyodysenteriae caecal colonisation.

\section{Materials and methods}

\section{Mice}

Male and female $\mathrm{C} 3 \mathrm{H} / \mathrm{HeJ}$ (lipopolysaccharide hyporesponsive) and $\mathrm{C} 3 \mathrm{H} / \mathrm{HeOuJ}$ (lipopolysaccharide responsive) mice aged 12-24 weeks were used in this study. These animals were obtained from breeder colonies maintained by the Department of Laboratory Animal Resources, Iowa State University, and were cared for in accordance with the guidelines stipulated by the Animal Care and Use Committee of that institution. Breeding colonies were established with mice originally procured from Jackson Laboratory, Bar Harbor, Maine, USA, and were routinely screened and found to be negative for the presence of serum antibodies to Sendai virus, murine hepatitis virus and Mycoplasma spp.

\section{Diet}

Breeding colonies and half of the mice used in these experiments were maintained on Mouse Lab Chow 5010 (Purina Mills Inc., St Louis, MO, USA), subsequently referred to as conventional rodent chow (CRC). Seventy-two hours before inoculation half of the mice were fed Teklad Diet 85420 (Harlan Sprague Dawley, Madison, WI, USA), subsequently referred to as Teklad diet (TD), and were maintained on that diet for the remainder of the experimental period. The approximate composition of TD 85420 is as follows (w/w): dextrose, $63.4 \%$; egg-white solids, $20.0 \%$; corn oil, $10.0 \%$; cellulose, $3.0 \%$; micronutrients as required. Food and water were given ad libitum.

\section{Bacteria and infection procedure}

Mice were infected with $S$. hyodysenteriae strain B204, serotype 2 , which was grown as previously described at $37^{\circ} \mathrm{C}$ in Trypticase Soy Broth (BBL Microbiology Systems, Cockeysville, MD, USA) supplemented with horse serum (HyClone Laboratories, Logan, UT, USA) $5 \%$ yeast extract (BBL) $0.5 \%$, L-cysteine (Sigma) $0.05 \%$ and VPI salt solutions $1 \%$ [8]. Log phase cultures of $S$. hyodysenteriae (motility 75-90\%) were evaluated for bacterial concentration with a PetroffHauser counting chamber. Mice were given $1 \times 10^{8}$ bacteria in $c .0 .5 \mathrm{ml}$ of culture broth intragastrically, twice within a 24-h period; food was withheld for $6 \mathrm{~h}$ before and $6 \mathrm{~h}$ after each of the two infections.

\section{Experimental design}

In the first experiment, mice were randomly allotted to one of the four treatment groups arising from the factorial arrangement of two diets and two mouse strains, with a total of 25 mice allotted to each group. Five randomly selected mice from each group were killed and examined by necropsy 1, 2, 4, 9 and 17 days after the second gastric intubation and utilsed for pathological examination. A second experiment, which was similar in design to the first, was performed for bacteriological studies. In that experiment, there were 20 mice/group, with four mice killed for necropsy at each of the same time points. Male and female mice were equally represented within treatment groups in both experiments.

\section{Pathological examination}

In the first experiment, caeca were evaluated grossly for atrophy, oedema and increased mucus in the ingesta, given a gross lesion score $(0-3$ : $0=$ normal, $3=$ severe caecal lesion) and then placed in neutral buffered formalin $10 \%$ until the end of the experimental period. After fixation, caeca were sectioned longitudinally, routinely processed, and embedded in paraffin. Sequential sections $(5 \mathrm{~mm})$ were stained with haematoxylin and eosin or Warthin-Starry (silver) stains. Specimens were randomly assigned an identification code number to ensure objectivity in evaluation of histopathological changes. Parameters evaluated microscopically for each specimen and the range of possible scores to be assigned to them were gland depth (2-7), lamina proprial cellular infiltrate (0-3), submucosal and lamina proprial oedema (0-3), superficial epithelial cell necrosis and loss $(0-3)$ and the presence or absence of spirochaetes on Warthin-Starrystained sections ( + or - ). A total lesion index score was arrived at by summation of all numerical lesion scores for each specimen.

\section{Bacteriological examination}

In the second experiment, aseptically removed caeca were weighed and suspended 1 in $100(\mathrm{w} / \mathrm{v})$ with phosphate-buffered saline $(136.9 \mathrm{mM} \mathrm{NaCl}, 8.1 \mathrm{mM}$ $\mathrm{Na}_{2} \mathrm{HPO}_{4}, 1.5 \mathrm{mM} \mathrm{KH} \mathrm{PO}_{4}, 2.7 \mathrm{mM} \mathrm{KCl}, \mathrm{pH}=7.2$ ) in sterile WhirlPak bags (Baxter Co., Minneapolis, 
MN, USA) and then homogenised in a stomacher laboratory blender (A. J. Seward Co., London). Appropriate serial dilutions of the homogenate were added to $5 \mathrm{ml}$ of molten $\left(45^{\circ} \mathrm{C}\right)$ trypticase soy agar containing ovine blood $5 \%$ and antibiotics selective for $S$. hyodysenteriae. This mixture was poured into $60-$ $\mathrm{mm}$ petri dishes and incubated anaerobically at $37^{\circ} \mathrm{C}$ for 72-96 h [13]. Zones of $\beta$-haemolysis were counted and used to calculate the number of cfu/caecum. Selected colonies were examined by dark-field microscopy to verify the presence of large, motile spirochaetes.

\section{Statistical analysis}

Numerical data from individual mice were used in analysis of variance procedures in SAS to obtain estimates of overall experimental error and to allow comparison of means of different groups.

\section{Results}

\section{Pathological parameters}

Total lesion index scores indicated that, throughout the experimental period, mice fed TD developed significantly more severe lesions than the mice fed CRC (Fig. 1). Mice fed TD also demonstrated an earlier onset and prolonged persistence of those more severe lesions in comparison to mice fed CRC (Fig. 2). On days 2 and 4, lesion severity was essentially the same for the two dietary groups. However, on all other days, there was a significant difference in lesion scores

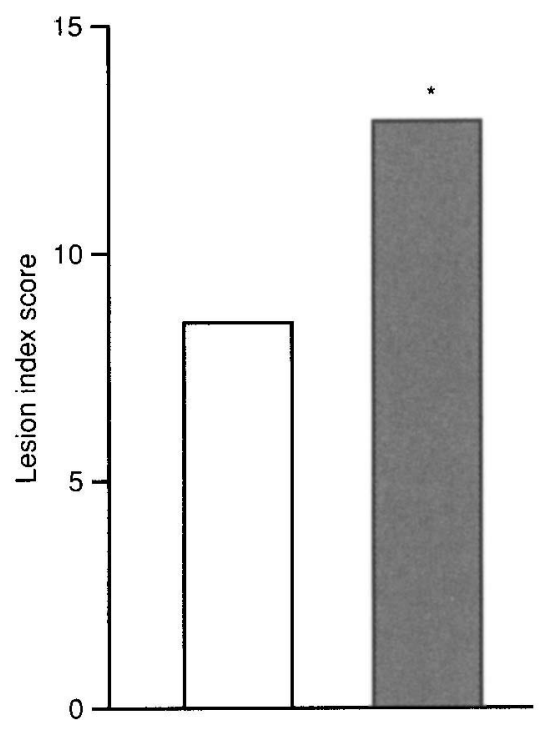

Fig. 1. The effect of Teklad diet 85420 (TD, $\square$ ) compared to conventional rodent chow $(\square)$ on gross and microscopic pathological parameters. Total lesion index score is the sum of scores for gross lesions, caecal gland depth, submucosal and lamina proprial oedema, lamina proprial cellular infiltrate and epithelial erosions. Mice fed TD had higher total lesion index scores, inclusive of strain, sex and day post-infection. ${ }^{*} \mathrm{p}<0.005$.

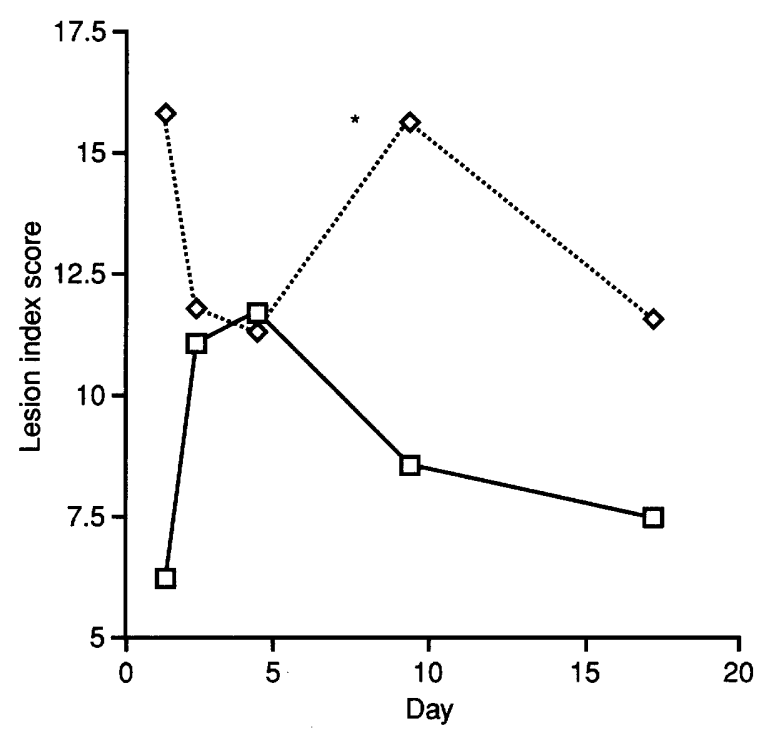

Fig. 2. The effect of Teklad diet 85420 (TD, - - $-_{--)}$ compared to conventional rodent chow (CRC, $-\square-$ ) on the temporal distribution of lesion occurrence in mice infected with $S$. hyodysenteriae. Lesion index score is the sum of all individual lesion scores for a particular treatment. Mice fed TD had more severe lesions that occurred much earlier and persisted longer than in mice fed CRC. ${ }^{*} \mathrm{p}<0.05$.

between the two groups, with the mice fed TD exhibiting more severe lesions.

\section{Microscopic observations}

Normal, uninfected mice, regardless of strain, diet or day post-infection, had caeca lined with a mucosa in which glands were two to three times as deep as they were wide (Fig. 3). The lamina propria was approximately one to a few cell layers thick and was occupied by spindle cells and occasional lymphocytes. Gutassociated lymphoid tissue (GALT), depending on sectioning, had few lymphoid follicles with small germinal centres (Fig. 4).

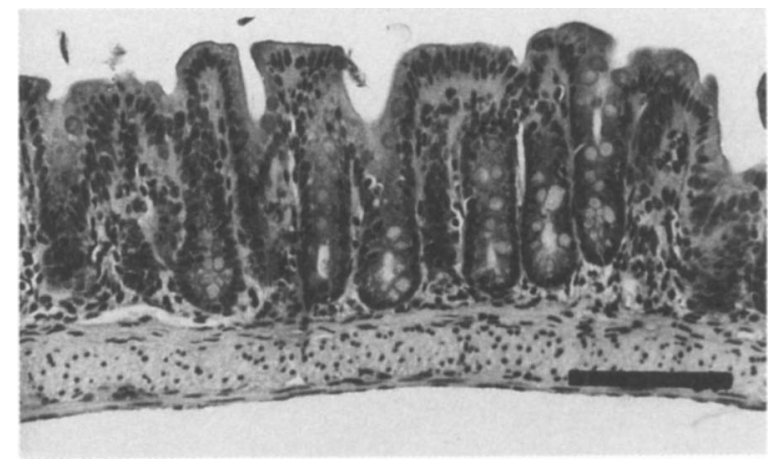

Fig. 3. Photomicrograph of caecum from a $\mathrm{C} 3 \mathrm{H} / \mathrm{HeOuJ}$ mouse fed TD. Note the relatively shallow glands, columnar superficial epithelial cells and indistinct lamina proprial and submucosal spaces of low cellularity. Stained with haematoxylin and eosin (HE). Bar = $100 \mu \mathrm{m}$. 


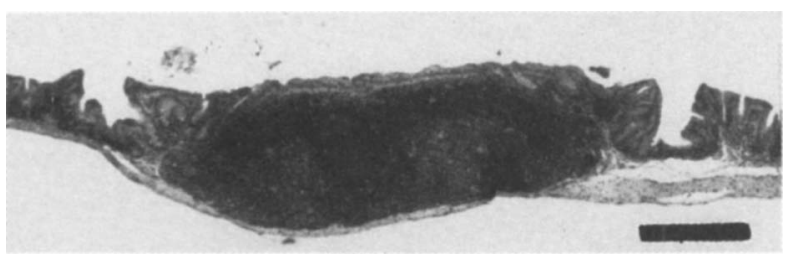

Fig. 4. Photomicrograph of gut-associated lymphoid tissue (GALT) in the caecum of a $\mathrm{C} 3 \mathrm{H} / \mathrm{HeOuJ}$ mouse fed TD. Note that this GALT nodule has few germinal centres and that the overlying caecal mucosa is thin. HE. $\mathrm{Bar}=300 \mu \mathrm{m}$

In mice infected with $S$. hyodysenteriae, severe caecal inflammation was characterised by superficial epithelial cell necrosis, lamina proprial and submucosal oedema, lamina proprial infiltration by inflammatory cells and colonic gland hyperplasia (Figs. 5-7). Other changes, such as dilatation of glands and exfoliation of necrotic epithelial and inflammatory cells into the

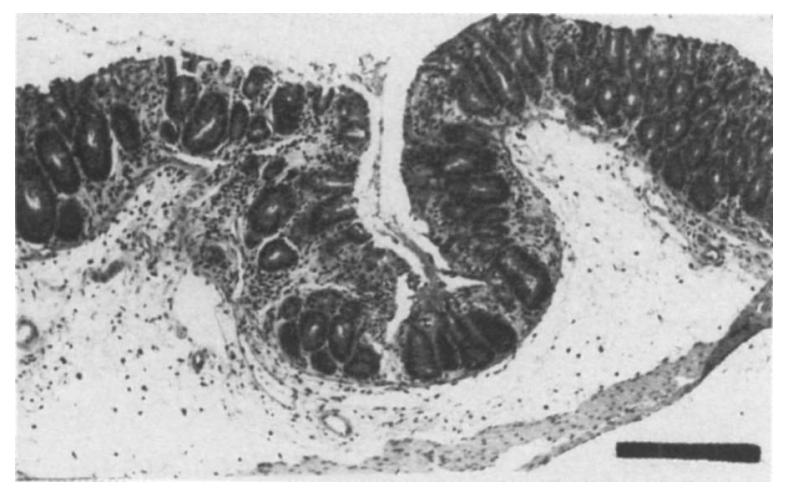

Fig. 5. Photomicrograph of caecum from a $\mathrm{C} 3 \mathrm{H} / \mathrm{HeOuJ}$ mouse fed TD, infected with $S$. hyodysenteriae and examined by necropsy $24 \mathrm{~h}$ later. Note the glandular dilatation and pronounced expansion of submucosal and lamina proprial spaces by oedema and inflammatory cells. HE. Bar $=200 \mu \mathrm{m}$.

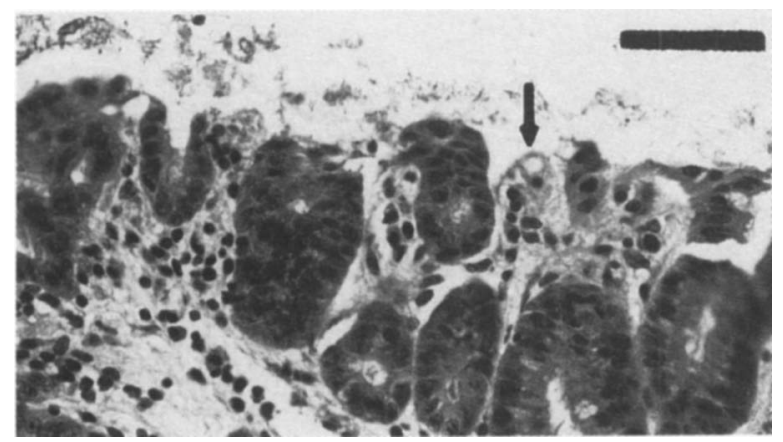

Fig. 6. Higher magnification of the superficial mucosa of the tissue shown in Fig. 5. Note the superficial epithelial cell loss (arrow), expansion of the lamina proprial space by oedema and inflammatory cells and crowding of dilated glands by tall, hyperplastic epithelial cells. HE. Bar $=50 \mu \mathrm{m}$.

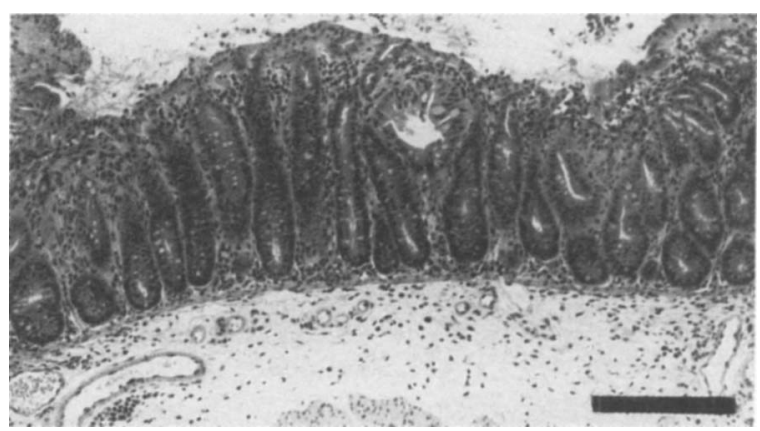

Fig. 7. Photomicrograph of caecum from a $\mathrm{C} 3 \mathrm{H} / \mathrm{HeOuJ}$ mouse fed TD, infected with $S$. hyodysenteriae and examined by necropsy 17 days later. Note the expansion of submucosal and lamina proprial spaces with oedema and inflammatory cells, superficial epithelial cell loss and lengthening of glands due to glandular epithelial hyperplasia. HE. $\mathrm{Bar}=200 \mu \mathrm{m}$.

caecal lumen, were variably present. Precise combinations of these features varied slightly from mouse to mouse and within individual caecal sections.

The microscopic features of severe lesions were the same, regardless of the treatment group from which the specimen derived. Comparison of severely affected specimens from each of the two diet treatment groups collected at different time points indicated that the morphological features typical of severe lesions, as described above, were present in both specimens (Figs. 5-7). As might be expected, gland hyperplasia was a more prominent finding in those animals infected for 17 days than in those necropsied 1 or 4 days after infection. Also, in animals examined after 17 days, GALT nodules were often grossly nodular because of the presence of prominent lympoid follicles with enlarged germinal centres (Fig. 8). These effects, gland and GALT hyperplasia, occurred in both diet treatment groups 17 days after infection.

In all but one of the mice infected with $S$.

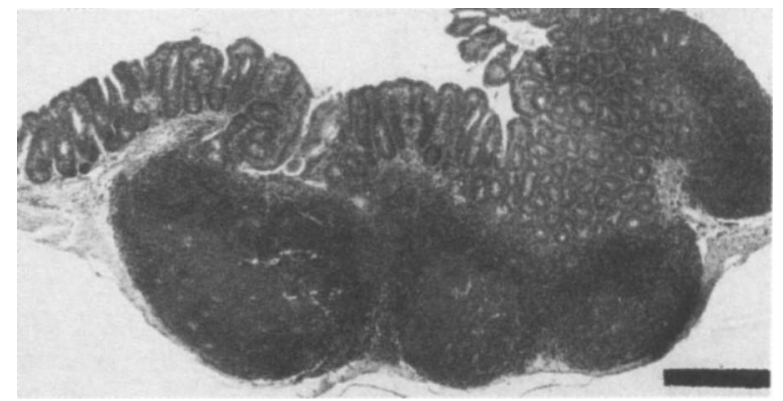

Fig. 8. Photomicrograph of caecum from a $\mathrm{C} 3 \mathrm{H} / \mathrm{HeOuJ}$ mouse fed TD, infected with $S$. hyodysenteriae and examined by necropsy 17 days later. Note that the caecal GALT has numerous germinal centres and is overlaid by a thickened, hyperplastic mucosa. HE. Bar $=300 \mu \mathrm{m}$. 
hyodysenteriae large spirochaetes were demonstrable within caecal lumina and glands of Warthin-Starry stained sections; intestinal spirochaetes were not demonstrated in the caeca of uninfected mice by this technique.

\section{Bacteriology}

There was no significant difference in cfu of spirochaetes isolated from animals as affected by strain, day or diet (Fig. 9). Animals on the TD had slightly higher mean cfu counts than those on CRC (7.61 and $7.36 \log _{10} \mathrm{cfu} /$ caecum, respectively), but the difference was not statistically significant. There was considerable animal to animal variation (range 6.3-8.4 $\log _{10}$ $\mathrm{cfu} /$ caecum).

\section{Discussion}

Two identical experiments were performed in these studies to compare the effects of TD and CRC in mice infected with $S$. hyodysenteriae with regards to the qualitative and temporal nature of lesion formation and numbers of $S$. hyodysenteriae in the caecum on various days after infection. Gross lesions observed in the bacteriology experiment were very similar to those seen in the experiment dedicated to lesion evaluation (data not shown).

Overall lesion severity, reflected as the total lesion index score, was significantly increased in mice fed

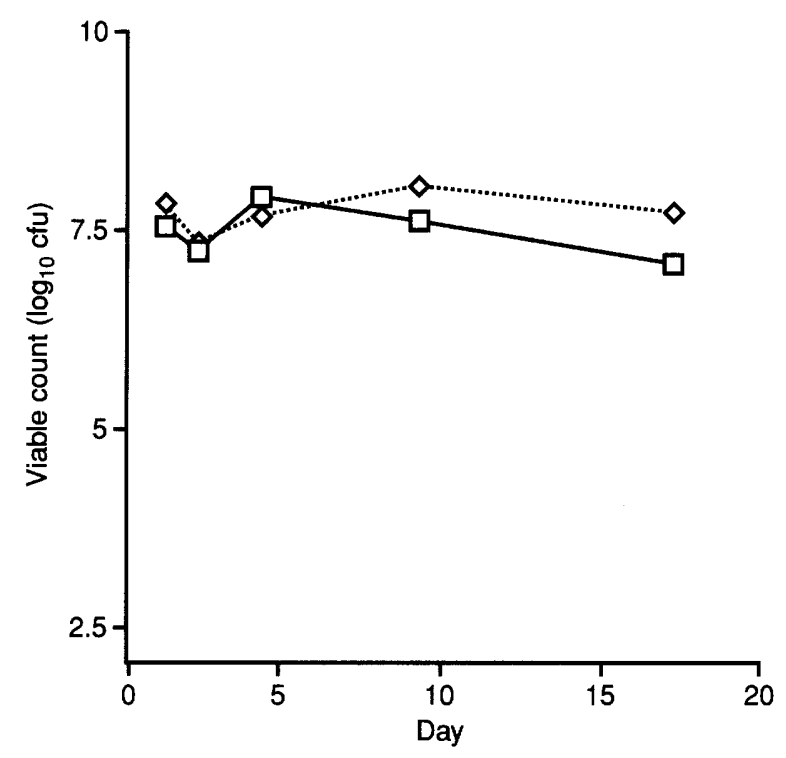

Fig. 9. A comparison of the effect of TD (- $\diamond_{--)}$and CRC (- $\square-)$ on colonisation of the murine caecum following infection with $S$. hyodysenteriae. Entire caeca were homogenised, diluted, added to molten blood agar containing antibiotics selective for $S$. hyodysenteriae and poured into plates. Haemolytic zones were counted and used to calculate the number of cfu. There were no significant differences between treatment groups.
TD. Furthermore, severe lesions consistently occurred within $24 \mathrm{~h}$ of $S$. hyodysenteriae infection in TD-fed mice. Importantly, the microscopic appearance of lesions of similar severity was not different between the two diet treatment groups. While hyperplasia of gland epithelium and GALT was present in mice from both diet treatment groups examined 17 days after infection, this expected effect is attributed to the duration of infection. In effect, the present studies establish that the use of TD in the murine model of $S$. hyodysenteriae infection results in caecal lesions that (a) have the same morphological features as those that occur with $\mathrm{CRC}$, and (b) occur at a much earlier time after infection (day 1) than previously reported.

In the first report describing the murine model of $S$. hyodysenteriae infection, mice were examined between 2 and 30 days after infection: gross caecal lesions and clinical signs (mucoid faeces) were seen throughout the experimental period [4]. In another report describing experimental infection of gnotobiotic mice, lesions occurred within 3 days in mice co-infected with $S$. hyodysenteriae and Bacteroides vulgatus [14]. While it has been shown that mice may remain colonised with $S$. hyodysenteriae for up to 70 days following infection, most workers examine experimentally infected mice between 10 and 20 days after infection $[5-8,11,12]$. As presented in this report, use of the Teklad 85420 diet increases the uniformity and usefulness of the murine model of $S$. hyodysenteriae infection by allowing earlier collection of caecal tissue samples with more consistent lesions from experimental animals.

One explanation for the marked differences between the treatment groups in this study might be that TD induces changes in the intestinal micro-environment that allow $S$. hyodysenteriae to maintain greater numbers, or exhibit increased viability or virulence, resulting in increased rapidity of lesion formation. These studies, in agreement with the previous report, refute the idea that increased numbers of spirochaetes account for the initial differences in lesion severity between the two groups of mice [12]. The lack of evidence supporting increased numbers of spirochaetes in the caeca of mice fed TD does not rule out the possibility that those that are present may exhibit enhanced virulence.

Other possible explanations for the more rapid onset of severe lesions in animals fed TD as compared to those fed CRC also relate to alteration of the intestinal environment by the diet, specifically the resident microflora. Early studies on the induction of swine dysentery in gnotobiotic pigs established that colonisation with $S$. hyodysenteriae alone was insufficient to elicit lesion production. Co-infection with other organisms, primarily gram-negative rods, such as Bacteroides vulgatus, is required for lesions to occur; the same requirements have been shown to be true in 
the murine model [14-18]. The role these other organisms play in the pathogenesis of swine dysentery is not known, but at least three possibilities seem to exist: (a) they result in the 'maturation' of the immune and inflammatory responses in the host; (b) they directly or indirectly induce or increase the virulence of $S$. hyodysenteriae: or (c) they produce cell-wall components or metabolites that, in synergy with or subsequent to expression of virulence factors by $S$. hyodysenteriae, result in lesion formation. If TD in some way selects for the increased growth or metabolic activity of other members of the caecal flora, either of the latter two mechanisms could explain the enhancement of acute lesion formation that occurs in infected mice fed that diet. In the original paper describing the effect of TD on the murine model of swine dysentery, Nibbelink reported increases in the numbers of caecal aerobic grampositive and gram-negative organisms, although the potentially more significant anaerobic cultures were not reported [12]. Those findings demonstrate that TD does, in fact, alter non-serpulinal microbial populations in the caecum.

It is also possible that the enhancing effect exerted by TD in this infection model is due to some direct effect on the host by the components of the diet, specifically the glucose and egg-white solids of which it is largely composed. Increased luminal glucose levels (20 mM) have been shown to interfere with maintenance of normal functional and structural properties of intestinal epithelial cell tight junctions [19, 20]. If mice fed TD experienced such alterations, mucosal damage following infection with $S$. hyodysenteriae might occur in an accelerated manner. As the present studies were designed exclusively to document the characteristics of the reported model, the mechanism by which TD effects enhanced susceptibility to $S$. hyodysenteriae cannot be determined from these studies.

Model disease systems in alternate animal hosts must meet several criteria in order to prove more useful than the true host species and therefore justify their use. They should have a predictable time course of lesion development such that the pathogenesis and its possible prevention can be studied. Lesion development should be acute and uniform, thereby allowing timely, accurate and useful experiments. Finally, the model should allow the development of pathological changes that are identical or very similar to those that occur in the true host species. The conventional murine model of $S$. hyodysenteriae infection meets some of these criteria with regards to swine dysentery. Use of TD in that murine model further meets those criteria by effecting acute onset of lesions that have identical morphological features to those that occur in mice fed conventional rodent chow. In addition, lesions occur much earlier and with greater uniformity in TD-fed mice, thereby providing the investigator with a more uniform and efficient model with which to study factors that influence development and resolution of lesions.

\section{References}

1. Harris DL, Glock RD, Christensen CR, Kinyon JM. Swine dysentery-I. Inoculation of pigs with Treponema hyodysenteriae (new species) and reproduction of the disease. Vet Med Small An Clin 1972; 67: 61-64.

2. Albassam MA, Olander HJ, Thacker HL, Turek JJ. Ultrastructural characterization of colonic lesions in pigs inoculated with Treponema hyodysenteriae. Can J Comp Med 1985; 49: 384-390.

3. Wilcock BP, Olander HJ. Studies on the pathogenesis of swine dysentery. I. Characterization of the lesions in colons and colonic segments inoculated with pure cultures or colonic content containing Treponema hyodysenteriae. Vet Pathol 1979; 16: $450-465$.

4. Joens LA, Glock RD. Experimental infection in mice with Treponema hyodysenteriae. Infect Immun 1979; 25: 757-760.

5. Joens LA, Glock RD, Kinyon JM. Differentiation of Treponema hyodysenteriae from $T$. innocens by enteropathogenicity testing in the CF1 mouse. Vet Rec 1980; 107: 527-529.

6. Suenaga I, Yamazaki T. Experimental Treponema hyodysenteriae infection of mice. Zentralbl Bakteriol Mikrobiol Hyg $A$ 1984; 257: $348-356$.

7. Nibbelink SK, Wannemuehler MJ. Effect of Treponema hyodysenteriae infection on mucosal mast cells and $\mathrm{T}$ cells in the murine cecum. Infect Immun 1990; 58: 88-92.

8. Nibbelink SK, Wannemuehler MJ. Susceptibility of inbred mouse strains to infection with Serpula (Treponema) hyodysenteriae. Infect Immun 1991; 59: 3111-3118.

9. Joens LA, Songer JG, Harris DL, Glock RD. Experimental infection with Treponema hyodysenteriae in guinea pigs. Infect Immun 1978; 22: 132-135.

10. Sueyoshi M, Adachi Y, Shoya S. Enteropathogenicity of Treponema hyodysenteriae in young chicks. Zentralbl Bakteriol Mikrobiol Hyg A 1987; 266: 469-477.

11. Mysore JV, Duhamel GE, Mathiesen MR. Morphologic analysis of enteric lesions in conventional and streptomycin-treated inbred $\mathrm{C} 3 \mathrm{H} / \mathrm{HeN}$ mice infected with Serpulina (Treponema) hyodysenteriae. Lab Anim Sci 1992; 42: 7-12.

12. Nibbelink SK, Wannemuehler MJ. An enhanced murine model for studies of Serpulina (Treponema) hyodysenteriae pathogenesis. Infect Immun 1992; 60: 3433-3436.

13. Kunkle RA, Kinyon JM. Improved selective medium for the isolation of Treponema hyodysenteriae. J Clin Microbiol 1988; 26: $2357-2360$.

14. Joens LA, Robinson IM, Glock RD, Matthews PJ. Production of lesions in gnotobiotic mice by inoculation with Treponema hyodysenteriae. Infect Immun 1981; 31: 504-506.

15. Meyer RC, Simon J, Byerly CS. The etiology of swine dysentery. I. Oral inoculation of germ-free swine with Treponema hyodysenteriae and Vibrio coli. Vet Pathol 1974; 11: $515-526$.

16. Meyer RC, Simon J, Byerly CS. The etiology of swine dysentery II. Effect of a known microbial flora, weaning and diet on disease production in gnotobiotic and conventional swine. Vet Pathol 1974; 11: 527-534.

17. Harris DL, Alexander TJ, Whipp SC, Robinson IM, Glock RD, Matthews PJ. Swine dysentery: studies of gnotobiotic pigs inoculated with Treponema hyodysenteriae, Bacteroides vulgatus, and Fusobacterium necrophorum. J Am Vet Med Assoc 1978; 172: 468-471.

18. Hayashi T, Suenaga I, Komeda T, Yamazaki T. Role of Bacteroides uniformis in susceptibility of Ta:CF\# 1 mice to infection by Treponema hyodysenteriae. Int $J$ Med Microbiol 1990; 274: 118-125.

19. Pappenheimer JR. Physiological regulation of transepithelial impedance in the intestinal mucosa of rats and hamsters. $J$ Membr Biol 1987; 100: 137-148.

20. Madara JL, Pappenheimer JR. Structural basis for physiological regulation of paracellular pathways in intestinal epithelia. $J$ Membr Biol 1987; 100: 149-164. 